\title{
Comparison of the outcome of transverse and circumferential capitonnage in surgical treatment of pulmonary hydatid cyst - a single centre study
}

\author{
Farooq Ahmad Ganie ${ }^{1^{*}}$, Masarat-ul Gani ${ }^{2}$, Khan M Yaqoob ${ }^{1}$, Syed Mohsin Manzoor ${ }^{1}$, G N Lone $^{1}$, \\ Abdual Majeed Dar ${ }^{1}$, Mohd Akbar Bhat ${ }^{1}$, Mudasir Hamid Bhat ${ }^{3}$ \\ ${ }^{1}$ Department of Cardiovascular and Thoracic Surgery, Sher-i-Kashmir Institute of Medical Sciences, Soura, \\ Srinagar, J \& K, India, ${ }^{2}$ Department of pathology GMC Srinagar, India; ${ }^{3}$ Department of Radiology, Sher-i- \\ Kashmir Institute of Medical Sciences, Soura, Srinagar, J \& K, India
}

\begin{abstract}
Background and objectives: The enucleation of the pulmonary hydatid cyst is followed by individual closure of bronchial air leaks and obliteration of the residual pericystic cavity by capitonnage, either by circumferential or interrupted transverse suture. The objective of the study was to compare the surgical outcome of transverse and circumferential capitonnage in terms of postoperative recovery course, residual cavitations, air leaks, cavitatory or pleural collections and the recurrence of primary disease after enucleation of the pulmonary hydatid cyst.
\end{abstract}

Methods: Patients with pulmonary hydatid cyst were included in the study and divided into two groups. Each group consisted of 30 patients. Patients of Group-1 underwent enucleation of the hydatid cyst followed by closure of bronchial air leaks with classical circumferential closure of the cavity and patients of Group-2 had enucleation of the hydatid cyst and closure of the cavity by transverse capitonnage.

Results: Ten cases (33.33\%) of Group-1 had hospital stay for more than 5 days compared to $4(13.33 \%)$ in Group-2 ( $p=0.03)$. Out of 30 patients who had undergone circumferential closure of the hydatid cavity, 5 (16.67\%) patients had residual cavitatory fluid collection while there was none in the other group. In Group-1, 7 (23.3\%) cases had reactionary intrapleural fluid collection compared to $2(6.6 \%)$ in Group-2 ( $p=0.035)$. After 3 months of follow-up, 4 patients in circumferential capitonnage had mild haemoptysis and 1 had aspergilloma while no such complication occurred in any patient in the transverse capitonnage group. No recurrence of cyst occurred in any case in both groups.

Conclusion: There was a considerable advantage of transverse capitonnage of the hydatid lung cavity after enucleation in terms of short hospital stay, minimal or no reactionary intrapleural or intra cavitatory collections and less air leaks.

IMC J Med Sci 2021; 15(2): 002

\section{Introduction}

Pulmonary cystic echinococosis, is a zoonosis cause by the larvae of the dog tapeworm Echinococcus granulosus and is considered as a major public health problem in cattle rearing countries with suboptimal hygiene and poor quality of lifestyle $[1,2]$. Chest pain and cough are the most prominent symptoms in young patients and those present early [3]. Most of the hydatid lung cases are diagnosed incidentally on a routine $\mathrm{X}$-xay

*Correspondence: Farooq Ahmad Ganie, Department of Cardiovascular and Thoracic Surgery, Sher-i-Kashmir Institute of Medical Sciences, Soura, Srinagar-190011, J \& K, India. E-mail: farooq.ganie@yamil.com 
examination. The majority of patients remain asymptomatic until the size of cyst increase to the level to cause mass effect [4] or the cyst becomes ruptured or infected leading to cough,chest pain, haemoptysis, or vomiting $[1,4]$. Haemoptysis is not uncommon presentation of pulmonary hydatid disease [5]. Massive haemoptysis (more than $600 \mathrm{~mL} /$ day) can occur.

Four species of Echinococcus produce infection in humans. E. granulosus and E. multilocularis are the most common, causing cystic echinococcosis and alveolar echinococcosis respectively $[4,2]$. The two other species, E. vogeli and E. oligarthrus, cause polycystic echinococcosis but have only rarely been associated with human infection [6]. The geographic distribution and animal host species vary by Echinococcus species, and mixed infections involving more than one species have been reported. In addition, different strains within an Echinococcus species may have variable morphology, genetic characteristics, infectivity to humans, and pathogenecity [6]. In endemic rural areas, prevalence rates of 2 to 6 percent or higher have been recorded [4,7]. New echinococcal infections continue to occur throughout life and increases with age $[1,6]$.

Hydatid cyst is usually filled with fluid. The inner layer is the germinative layer that produces the hydatid fluid and the secondary cysts which may develop into daughter cysts and develops within the native cyst separately [8]. After at least 10 to 12 months following infection, protoscolices are produced within the brood capsules. Cysts may contain liters of fluid and thousands of protoscolices. The cysts which are containing protoscolices are fertile and can reproduce daughter cysts, whereas cysts without protoscolices are sterile $[6,8]$. The outer layer is an acellular, laminated membrane which is of variable thickness. A host reaction occurs around this membrane [8]. The natural course of hydatid cyst is progressive growth over a long period. Hydatid cyst compresses bronchial structures and produce pulmonary atelectasis accompanied by pneumonia [3]. Diagnosis is made by imaging evaluation (Chest $\mathrm{X}$-ray or CT scan), and supported by serology in the majority of cases $[4,7]$. Chest radiography provides a correct diagnosis in about $99 \%$ of patients [9].
Surgery remains the treatment of choice in hydatid lung disease $[2,3,10]$ which includes either the cystotomy or cystotomy with capitonnage [11]. The aim of surgery remains the total excision of the cyst and obliteration of the residual cavity [7]. The enucleation is followed by individual closure of bronchial air leaks and obliteration of the residual cavity by capitonnage [12] either by circumferential or by interrupted transverse capitonnage. However, no scientific consensus over selection of these techniques has been reached till now. The site and the size of the cyst usually dictate the type of procedure to be used [12]. Pulmonary resection is the usual surgical procedure in giant and large pulmonary hydatid cysts $[6,13]$. Giant hydatid cysts of the lung (diameter, $\geq 10 \mathrm{~cm}$ ) are considered more difficult to treat surgically than are smaller cysts [13].

The aim of the present study was to compare the surgical outcome of transverse and circumferential capitonnage after enucleation of the pulmonary hydatid cyst.

\section{Materials and methods}

The study was conducted in the Department of Cardiovascular and Thoracic surgery, Sher-iKashmir Institute of Medical Sciences, Soura, Srinagar, India from December 2013 to December 2019. The study was approved by the Institutional Ethical Board. Informed consent was obtained from all patients prior to the enrolment into the study. The study included patients with pulmonary (parenchymal) hydatid cyst. All the patients had unruptured and uncomplicated pulmonary hydatid cysts without any previous surgical history for the same disease. The mean dimension of the cystic lesion at presentation was $12 \times 15 \mathrm{~cm}$ involving any lobe of either of the two lungs. Patients with ruptured cysts and those with a concomitant pleural effusion with intact cyst were excluded from the study. Enrolled patients were divided into two groups namely Group-1 and Group-2. Each group consisted of 30 patients. Group-1 patients underwent enucleation of the hydatid cyst followed by closure of bronchial air leaks, and circumferential capitonnage starting from the base of the cyst causing centripetal collapse of the residual cavity. Patients of Group-2 underwent closure of the cavity by interrupted transverse 
apposition causing the coplanar collapse of the corresponding pericyst walls after enucleation of the hydatid cyst. The transverse single suture apposition included 1 to $1.5 \mathrm{~cm}$ horizontal silk sutures at a separation distance of 1.5 to $2 \mathrm{~cm}$ in the coplanar areas of the pericyst and less than that in the areas of crevices.

Patients were observed for postoperative recovery course, character and volume of the fluid from postoperative chest drainage tube, any residual cavitations or collection, any air leak and its duration and any subsequent complications.

\section{Results}

Detail comparison of post operative outcome of Group-1 and 2 patients is shown in Table-1. Ten cases (33.33\%) of Group-1 had hospital stay for more than 5 days compared to $4(13.33 \%)$ in Group$2(p=0.03)$. Out of 30 patients who had undergone circumferential closure of the hydatid cavity, 5 $(16.67 \%)$ patients had residual cavitatory fluid collection that either required prolonged postoperative antibiotic treatment or an image guided percutaneous drainage or maintenance of chest tube for more than a week (Table-1). Of the 5 patients, 2 were managed conservatively for the residual collection whereas 2 cases required image guided percutaneous pigtail drainage. None of these 3 patients required immediate reexploration. No residual cavity was observed in patients in Group-2. In Group-1, 7 (23.3\%) cases had reactionary intrapleural fluid collection compared to $2(6.6 \%)$ in Group-2 $(p=0.035)$. After 3 months of follow-up, 4 patients who underwent circumferential capitonnage had mild haemoptysis while no such complication occurred in any patient in the transverse capitonnage group (Group-2). One patient in the circumferential capitonnage group had a significant residual cavitatory lesion and had aspergilloma and presented with subtle haemoptysis. This patient required a surgical intervention for decortication and obliteration of the cavity. Coincidently, this patient had the largest of the hydatid cysts amongst all the study patients suggesting the likelihood of failure of circumferential suturing to obliterate the cavity completely either due to non apposition of pericyst walls or a suture cut-through. Rest of the four patients had their cavities obliterated on 3 month follow-up. No recurrence of cyst occurred in any case in both groups.

Table-1: Comparison of outcome of transverse and circumferential capitonnage method

\begin{tabular}{|c|c|c|}
\hline \multirow[b]{2}{*}{ Outcome } & \multicolumn{2}{|c|}{ Capitonnage method } \\
\hline & $\begin{array}{c}\text { Circumferential } \\
\begin{array}{c}(\mathrm{N}=30) \\
\mathrm{n}(\%)\end{array}\end{array}$ & $\begin{array}{c}\text { Transverse } \\
\begin{array}{c}(\mathbf{N}=30) \\
n(\%)\end{array}\end{array}$ \\
\hline $\begin{array}{l}\text { Hospital stay } \\
>5 \text { days* }\end{array}$ & $10(33.3)$ & $4(13.3)$ \\
\hline Residual cavity & $5(16.7)$ & 0 \\
\hline Air leak mild & $6(20)$ & $3(10)$ \\
\hline $\begin{array}{l}\text { Reactionary } \\
\text { intrapleural fluid } \\
\text { collection** } \\
\text { Events after } 3 \mathrm{~m}\end{array}$ & $7(23.3)$ & $2(6.6)$ \\
\hline Haemoptysis & $4(13.3)$ & 0 \\
\hline Aspergilloma & $1(3.3)^{* * *}$ & 0 \\
\hline $\begin{array}{l}\text { Recurrence of } \\
\text { primary disease }\end{array}$ & 0 & 0 \\
\hline $\begin{array}{l}\text { Note: }{ }^{*} p=0.03 \text { and } \\
\text { Gr-1 (Circumferenti } \\
* * * \text { Required surqic }\end{array}$ & $\begin{array}{l}{ }^{*} p=0.035 \text { comp } \\
\text { and Gr-2 (Trans } \\
\text { intervention. } m\end{array}$ & $\begin{array}{l}d \text { between } \\
\text { rse); }\end{array}$ \\
\hline
\end{tabular}

\section{Discussion}

Pulmonary hydatid cyst, a zoonotic disease, is frequently encountered in cattle raising regions of the world and has been observed most often in the Asiatic countries $[1,4,8]$. Humans act as an accidental intermediate hosts and harbour cysts, which are most commonly found in the liver and lung but can be discovered in nearly any organ [1]. Nearly one quarter of the patients is asymptomatic. The most predominant complaints of the patients were chest pain followed by unproductive cough, haemoptysis and fever $[1,4]$. Surgery is the primary modality of treatment for patients with pulmonary hydatid disease $[1,11]$. The capitonnage method has more advantages in surgery of uncomplicated pulmonary hydatid cysts $[13,14]$. Some studies demonstrated a low complication rate associated with removing the thin margins of the residual cavity and changing the shape of it into a "saucer." The results were satisfactory and 
comparable to the results of other studies on pulmonary hydatid cysts [12]. Also, it has been proven that capitonnage results in less postoperative morbidity [15] as compared to deroofing of the cyst cavity. The capitonnage is the best way to tackle the cyst cavity and is definitely better then cystotomy because it reduces the prolonged air leak [16] and hospital stay. However, not all the studies favour the capitonnage [17].

We used standard posterolateral or lateral thoracotomy in cases of pulmonary hydatid cyst surgery. In this study, 30 patients underwent enucleation of the cyst with closure of the bronchial opening with circumferential capitonnage (conventional capitonnage). We found higher rates of early complications like post operative air leak, reactionary plural collection and residual cavity in patients of this group. Also, there were more delayed complications among patients of circumferential capitonnage group like mild haemoptysis and aspergilloma.

In our 30 other patients in Group-2, interrupted transverse capitonnage was done after enucleation of the cyst and closure of any bronchial air leaks. The operation was done by same group of surgeons. Almost $100 \%$ of the patients had immediate cure. There was no major fatality. In transverse capitonnage procedure, it was not mandatory to do approximation and suturing the cavity edges tightly because the pulmonary parenchyma obliterates the space as the side of cavity is covered by pleura. Capitonnage provides complete obliteration of the pericystic cavity to prevent air leak from residual bronchial openings and removes any potential space for fluid collection [18]. Without capitonnage, the wall of the pericystic cavity becomes covered by epithelial cells which takes an uncertain length of time $[1,15]$ Capitonnage, though prolongs the operation time, there is no report of any adverse implication. Our study strongly suggests the effectiveness of interrupted transverse capitonnage as there was not a single case of residual cavity or fluid collection in any of the 30 patients who had undergone interrupted transverse closure of the hydatid cavity. The intrapleural drains were removed within 3 to 4 days. Two patients had mild reactionary plural collection and none had delayed complications like haemoptysis and aspergilloma.

Therefore, we concluded that post enucleation, the large and deep parenchymal hydatid pericyst cavities should be obliterated completely and preferably with interrupted transverse capitonnage leaving least chances for residual cavities and the complications thereof. Interrupted transverse capitonnage is undoubtedly the ideal way of obliteration and closure of residual cavity after enucleation of both large and small hydatid lung lesions with minimal or no complications, both immediate and remote. This method ensures complete apposition and hence effective obliteration of the large cavities. The length, depth and the distance between the corresponding sutures has to be individualised depending upon the shape and size of the hydatid cavity and the integrity of the pericyst. However, further studies with large number of cases need to be done to assess late complications.

\section{Source of Funding: Nil.}

\section{Conflict of interest: nil}

\section{References}

1. Turna A, Yılmaz MA, Hacribrahimoğlu G, Kutlu CA, Bedirhan MA. Surgical treatment of pulmonary hydatid cysts: is capitonnage necessary? Ann Thorac Surg. 2002; 74: 191-195.

2. Goni, MO, Karim F, Alam MK, Chowdhury GMA, Ahsan S. Capitonnage versus noncapitonnage surgery for pulmonary hydatid cyst: a head to head study. J Dhaka Med Coll. 2015; 23(1): 94-101.

3. Kabiri H, Traibi A, Hammoumi ME, Oueriachi FE, Arsalane A. Parenchyma sparing procedures is possible for most pulmonary hydatid disease without recurrence and low complications. Med Arch. 2012; 66(5): 332-335

4. Santivanez S, Garcia HH. Pulmonary cystic echinococcosis. Curr Opin Pulm Med. 2010; 16(3): 257-261. 
5. Ayed AK, Alshawaf E: Surgical treatment and follow-up of pulmonary hydatid cyst. Med Princ Pract. 2003; 12(2): 112-116.

6. Garg MK, Sharma M, Gulati A, Gorsi U, Aggarwal AN, Agarwal R, Khandelwal N. Imaging in pulmonary hydatid cysts. World J Radiol. 2016; 8(6): 581-587.

7. Aldahmashi M, Alassal M, Kasb I, Elrakhawy H. Conservative surgical management for pulmonary hydatid cyst: analysis and outcome of 148 cases. Can Respir J. 2016; 2016: 8473070 .

8. Moro P, Schantz PM. Echinococcosis: a review. Int J Infect Dis. 2009; 13(2): 125-33.

9. Ahmad M, Khan SA, Shah SZ, Nazar HS, Bilal A, Khan MA, Bahauddin, Fazeel H. Effect of size on the surgical management of pulmonary hydatid cyst: I Ayub Med Coll Abbottabad. 2014; 26(1): 42-45.

10. Nabi MS, Waseem T. Pulmonary hydatid disease: what is the optimal surgical strategy? Int J Surg. 2010; 8(8):612-616.

11. Nabi MS, Waseem T, Tarif N, Chima KK. Pulmonary hydatid disease: is capitonnage mandatory following cystotomy? Int J Surg. 2010; 8(5): 373-376.

12. Bilgin M, Oguzkaya $F$, Akçali $Y$. Is capitonnage unnecessary in the surgery of intact pulmonary hydatic cyst? ANZ J Surg. 2004; 74(1-2): 40-42.
13. Burgos R, Varela A, Castedo E, Roda J, Montero CG, Serrano S, Téllez G, Ugarte J. Pulmonary hydatidosis: surgical treatment and follow-up of 240 cases. Eur J Cardiothorac Surg. 1999; 16(6): 628-634.

14. Sokouti M, Golzari SE, Aghdam BA. Surgery of uncomplicated pulmonary hydatid cysts: capitonnage or uncapitonnage? Int I Surg. 2011; 9(3): 221-224

15. Durakbasa CU, Sander S, Sehiralti V, Tireli GA, Tosyali AN, Mutus M. Pulmonary hydatid disease in children: outcome of surgical treatment combined with perioperative albendazole therapy. Pediatr Surg Int. 2006; 22(2): 173-178.

16. Kosar A, Orki A, Haciibrahimoğlu G, Kiral H, Arman B. Effect of capitonnage and cystotomy on outcome of childhood pulmonary hydatid cysts. J Thorac Cardiovasc Surg. 2006; 132(3): 560-564.

17. Erdogan A, Ayten A, Demircan A. Methods of surgical therapy in pulmonary hydatid disease: is capitonnage advantageous? ANZ J Surg. 2005; 75(11): 992-996.

18. Mahmodlou R, Sepehrvand N, Nasiri M. Saucerization: a modified uncapitonnage method of surgery for pulmonary hydatidosis. World J Surg. 2013; 37(9): 2129-2133. 